\section{Profilaxias na gestação}

\section{Théo Fernando Bub}

\section{1 - Profilaxia secundária da febre reumática}

O Brasil ainda apresenta uma frequência alta de febre reumática $(\mathrm{FR})$, mais prevalente no sexo feminino, e que pode se manifestar apenas como uma doença reumatológica ou associada a manifestações cardíacas. Mesmo as formas leves de cardite podem resultar em doença orovalvular grave. Os agentes etiológicos são os estreptococos beta-hemolíticos do grupo $\mathrm{A}(\mathrm{GABHS})$, responsáveis por $10 \%$ das faringites ${ }^{1}$.

Toda mulher com história de FR, apresentando ou não cardite, deve ser orientada para o risco da recidiva. A antibioticoterapia profilática deve ser continuada durante a gestação, conforme os seguintes critérios:

3.1.1 - FR com história de cardite e/ou lesão valvular: até os 40 anos

3.1.2 - FR com história de cardite e sem lesão valvular: até 10 anos após o surto de FR

3.1.3 - FR sem história de cardite e sem lesão valvular: por cinco anos após o surto reumático ou até os 21 anos de idade $^{2}$ (IB)

O antibiótico recomendado é a penicilina benzatina (Benzectacil $^{\circledR}$ ) na dosagem de 1.200.000 UI, via IM profunda a cada três semanas. Na impossibilidade do esquema anterior, apesar de ser menos eficaz que a apresentação injetável, pode ser utilizada a penicilina $\mathrm{V}$ potássica $\mathrm{VO}$, na dose de 400.000 UI (250 mg) a cada 8 ou 12 horas. Em casos de alergia à penicilina, está indicado o estearato de eritromicina (Pantomicina $^{\circledR}$ ), na dose de $250 \mathrm{mg}$ VO a cada 12 horas.

Vacinas antiestreptocócicas estão contraindicadas em pacientes com antecedentes de FR, pois a presença de proteína $M$ nas vacinas pode desencadear a doença reumática $\operatorname{aguda}^{3}$ (IIIB).

\section{2 - Profilaxia da endocardite infecciosa}

Procedimentos diagnósticos ou terapêuticos podem resultar em bacteremia e levar à El durante a gestação, estando incluídos procedimentos dentários, geniturinários e gastrointestinais ${ }^{4}$. O parto também está associado a situações que favorecem a bacteremia, tais como: rotura de membranas por mais de 6 horas, remoção manual da placenta ${ }^{5}$, trabalho de parto prolongado ${ }^{6}$ e baixo nível socioeconônico ${ }^{7}$. Apesar dessas constatações, não existe um consenso nas recomendações da profilaxia antibiótica. Os guidelines da ACC e AHA não recomendavam a profilaxia rotineira para parto cesáreo, nem para curetagem uterina e aborto terapêutico na ausência de infecção. E para o parto vaginal, a profilaxia era opcional nas pacientes de alto risco ${ }^{2}$. Em atualização publicada recentemente, a profilaxia não está sendo recomendada em parto vaginal, mas apenas para portadoras de condições cardíacas que representam maior risco de resultados adversos, caso desenvolvam El, e em situações de maior risco de bacteremia. Além disso, é salientado que a associação com comorbidades, como diabetes, imunossupressão e necessidade de diálise, representam fatores predisponentes adicionais que devem ser considerados ${ }^{8}$.

No entanto, publicações também recentes demonstraram incidência aumentada de bacteremia após trabalho de parto (variando de $14 \%$ a $19 \%$ ) e com germes que podem provocar El. Como tanto a mortalidade materna (22\%) como a fetal (15\%) estão elevadas na El durante o CGP ${ }^{9}$ a recomendação de Elkayam e cols. ${ }^{9}$ e Siu e cols. ${ }^{10}$ é a de que a profilaxia antibiótica fosse mantida na rotina antes do parto, tanto vaginal como cesáreo, em portadoras de lesões predisponentes, e que na prática seria esta a conduta adotada nos serviços especializados, independente das novas recomendações publicadas.

As lesões consideradas predisponentes seriam as descritas como de alto risco para El nos guidelines atuais do ACC e AHA, além das valvulopatias congênitas ou adquiridas (estenoses e insuficiências) e a miocardiopatia hipertrófica, conforme sugerido pelo National Institute for Health and Clinical Excellence (NICE) Guidelines, em março de $2008{ }^{11}$. Esse guideline, publicado na Inglaterra, não mais recomenda profilaxia em procedimentos dentários ou respiratórios, mas apenas para procedimentos gastrointestinais (GI) ou geniturinários $(G U)$, quando há suspeita de infecção préexistente. Como não existem evidências comprovando a eficácia da profilaxia, nem a segurança de sua suspensão, não há um consenso sequer entre os guidelines.

A Associação Americana de Urologia recomenda como rotina a profilaxia antibiótica periprocedimento em todas as cirurgias urológicas, a fim de reduzir infecções localizadas ou sistêmicas ${ }^{12}$.

Também é recomendada profilaxia antibiótica de rotina em parto cesáreo, tanto de urgência como eletivo, com a finalidade de reduzir a endometrite (em 2/3 a 3/4 dos casos) e a infecção na ferida operatória. Tanto a ampicilina como a cefalosporina de primeira geração são consideradas igualmente eficazes ${ }^{13}$. Além disso, o resultado de uma metanálise recomenda que a administração do antibiótico é mais eficaz quando realizada antes da incisão da pele, se comparada ao momento do clampeamento do cordão umbilical, e sem comprometer os resultados neonatais ${ }^{14}$.

Resumindo, as recomendações do Colégio Americano de Obstretrícia e Ginecologia (ACOG) para o uso rotineiro de profilaxia antibiótica (com ampicilina ou cefalosporina de primeira geração) antes de procedimentos obstétricos e ginecológicos são as seguintes:

- Pacientes submetidas a parto cesáreo;

- Histerectomia abdominal ou vaginal;

- Curetagem de sucção (em casos de aborto);

- Em casos de histerosalpingografia com trompas de falópio dilatadas (não indicado naquelas sem história de infecção pélvica).

Não recomendado por evidências insuficientes:

- Cerclagem cervical de emergência;

- Inserção de DIU em paciente sem doença inflamatória pélvica; 
- Laparoscopia diagnóstica.

Recomendações para a profilaxia da El (ampicilina ou amoxacilina + gentamicina):

- É opcional, em parto vaginal não complicado, nas pacientes cardiopatas consideradas de alto risco para El (próteses valvulares mecânicas e biológicas, El prévia, malformações cardíacas congênitas complexas cianóticas e enxertos sistêmico-pulmonares);

- É indicada em parto vaginal complicado, com infecção intra-amniótica nos casos descritos anteriormente;

- Poderiam se beneficiar da profilaxia da El, em alguns procedimentos cirúrgicos, as portadoras de cardiopatia estrutural de alto risco (descritas anteriormente) e as de risco moderado (valvulopatias adquiridas, incluindo estenoses e regurgitações, cardiopatias congênitas acianóticas e miocardiopatia hipertrófica) ${ }^{15}$.

Portanto, é rotina o uso de antibiótico profilático antes de procedimentos ou cirurgias urológicas, ginecológicas e obstétricas, sendo o antibiótico geralmente o mesmo indicado para a profilaxia da EI.

Bacteremia transitória é comum durante a manipulação dentária e do tecido periodontal (exodontia, cirurgia periodontal etc.), e também durante atividades diárias frequentes, como escovação, uso de fio-dental ou palito, e na mastigação de alimentos. Atualmente têm sido mais valorizadas como causadoras de $\mathrm{El}$ as exposições mais frequentes à bacteremia do que as esporádicas (associadas a procedimentos $)^{16}$. E entre os procedimentos dentários, são considerados de risco aqueles que provocam manipulação da gengiva, na região periapical dos dentes ou quando há perfuração da mucosa oral. A recomendação da ACC/AHA é que a profilaxia seja realizada em pacientes consideradas de alto risco ${ }^{8}(\mathrm{IlbB})$.

Se não houver um consenso entre a recomendação cardiológica e a conduta odontológica em relação à profilaxia da El, a Associação Americana de Odontologia sugere que os riscos e benefícios sejam expostos às pacientes e que seja obtido um consentimento informado definindo qual a conduta adotada ${ }^{16}$.

No Brasil, a cardiopatia reumática ainda é altamente prevalente, além de predominar em populações de menor nível socioeconômico, mais expostas a infecções. A recomendação desta diretriz é que seja realizada a profilaxia antibiótica nas lesões de alto risco e em situações de maior risco de bacteremia, conforme orientação do ACC e AHA, mas com a inclusão do parto. Como em partos cesáreos já é rotina obstétrica o uso de antibióticos profiláticos, seria apenas um ajuste de drogas, e resta a dúvida de qual seria a melhor conduta em parto vaginal na ausência de infecção. A orientação seria de avaliar individualmente os riscos, as dificuldades em conseguir assistência médica e decidir, em conjunto com a paciente, pela conduta mais segura (IlaC).

Seria recomendado também que se mantivesse a profilaxia antibiótica nas lesões consideradas de risco moderado (valvulopatias adquiridas, cardiopatias congênitas acianóticas e cardiomiopatia hipertrófica) no parto vaginal e cesáreo, até que estudos comprovem a segurança de sua retirada (IlaC).
As lesões cardíacas consideradas de alto risco para El e com a recomendação atual de profilaxia antibiótica, de acordo com o ACC e $\mathrm{AHA}^{8}$, são as seguintes:

1) Prótese valvar cardíaca (a mortalidade por El é $\geq 20 \%$ );

2) El prévia;

3) Cardiopatias congênitas (CC):

3.1 - CC cianóticas não corrigidas ou com procedimentos paliativos (shunts, derivações);

3.2 - CC corrigidas com enxertos de materiais protéticos. A profilaxia está indicada em procedimentos de risco durante o período da endotelização (6 meses);

3.3 - CC corrigida com permanência de defeito residual ao lado de enxerto com material protético (a permanência do defeito inibe a endotelização);

3.4 - Pacientes transplantadas que desenvolvem valvopatia $^{8}$.

As situações associadas à bacteremia e com indicação de profilaxia da El são:

1) Procedimentos dentários que provocam manipulação da gengiva, na região periapical dos dentes ou quando há perfuração da mucosa oral;

2) Pacientes com infecção gastrointestinal ou geniturinária ativa e que necessitam manipulação geniturinária não eletiva. A orientação é que devem ter cobertura antibiótica também para o enterococcus (com amoxacilina ou ampicilina);

3) Procedimentos em tecidos infectados, como pele, anexos e tecido músculo-esquelético, onde os germes passíveis de desenvolver El são o staphylococcus o e streptococcus $\beta$ hemolítico: o regime terapêutico deve conter antibiótico que atue nestes germes ${ }^{8}$.

\section{Esquemas de profilaxia ${ }^{8}$ :}

10) Para o parto ${ }^{9,10}$ : (IlaC)

- Ampicilina ou amoxacilina 2,0 g IV, mais gentamicina 1,5 $\mathrm{mg} / \mathrm{kg}$ IV (não exceder $120 \mathrm{mg}$ ) 30 minutos antes do parto; e 6 horas após, ampicilina ou amoxacilina 1,0 g IV ou VO.

$\left.2^{\circ}\right)$ Paciente alérgica à penicilina:

- Vancomicina 1,0 g IV, em infusão por até duas horas, mais gentamicina 1,5 mg/kg IV (não exceder 120 mg) 30 minutos antes do parto.

\section{Para os procedimentos dentários descritos ${ }^{8}$ (IIbC)}

$\left.1^{\circ}\right)$ Amoxacilina 2,0 g VO uma hora antes do procedimento;

$\left.2^{\circ}\right)$ Se está sem $\mathrm{VO}$ :

- Ampicilina 2,0 g IM ou IV 30 minutos antes do procedimento ou cefazolina ou ceftriaxone 1,0 g IM ou IV;

$\left.3^{\circ}\right)$ Se alérgica à penicilina ou ampicilina e para uso VO:

- Cefalexina 2,0 g uma hora antes do procedimento ou clindamicina $600 \mathrm{mg}$ ou azitromicina ou claritromicina $500 \mathrm{mg}$;

$4^{\circ}$ ) Se alérgica à penicilina ou ampicilina e sem VO:

- Cefazolina ou ceftriaxone 1g IM ou IV 30 minutos antes 
do procedimento ou clindamicina $600 \mathrm{mg}$ IM ou IV.

Se por alguma razão houver impossibilidade de administrar a dose prévia ao procedimento, administrar até duas depois do mesmo ${ }^{17}$.

Para as pacientes consideradas de risco moderado (valvulopatias adquiridas, cardiopatias congênitas acianóticas e cardiomiopatia hipertrófica):

$\left.1^{\circ}\right)$ Amoxacilina: 2,0 g VO uma hora antes do parto ou ampicilina 2,0 g IM ou IV 30 minutos antes do parto.

\section{Referências}

1. Snow V. Principles of antibiotic use for acute pharingitis in adults. Ann Intern Med. 2001; 134: 506-8.

2. ACC/AHA 2006 Guidelines for the management of patients with valvular heart disease: a report of the American College of Cardiology/American Heart Association Task Force on practice guidelines: developed in collaboration with the Society of Cardiovascular Anesthesiologists: endorsed by the cardiovascular angiography and interventions and the Society of Thoracic Surgeons. Circulation. 2006; 114: 84-231.

3. Dale JB. Group A streptococcal vaccines. Infect Dis Clin North Am. 1999; 13 (1): 227-43.

4. Ebrahimi R, Leung CY, Elkayam U, Reid CL. Infective endocarditis. In: Elkayam $\mathrm{U}$, Gleicher N. (editors.). Cardiac problems in pregnancy. 3rd ed. New York: Wiley-Liss; 1998. p. 191-8.

5. Sugrue D. Antibiotic prophylaxis against infective endocarditis after normal delivery: is it necessary? Br Heart J. 1980; 44: 489-502.

6. Tiassi CLD, Rodrigues OFF, Santos ARL, Franken RA, Mimica L, Tedesco JJA Bacteriemia induzida pelo trabalho de parto: cabe a profilaxia da endocardite infecciosa? Arq Bras Cardiol. 1994; 62: 91-4

7. Chenoweth C, Schaberg D. The epidemiology of enterococci. Eur J Clin Microb Infect Dis. 1990; 9: 80-9.

8. Wilson W, Taubert KA, Gewitz M, Lochart PB, Baddour LM, Levison M, et al. Preventive of infective endocarditis: guidelines from the American Heart Association Rheumatic Fever, Endocarditis and Kawasaki Disease Committee, Council Cardiovascular Disease in the Young, and the council on Clinical Cardiology, Council on Cardiovascular Surgery and Anesthesia, and the Quality of Care and outcomes Research Interdisciplinary Working Group. Circulation. 2007; 116 (15): 1736-54.

9. Elkayam U, Bitar F. Valvular heart disease and pregnancy. J Am Coll Cardiol. 2005: 46: 223-30. $2^{\circ}$ ) Quando alérgicas à penicilina:

- Vancomicina 1,0 g IV em infusão por até duas horas 30 minutos antes do parto $9,10,11,17$ (IlaC).

Todas as pacientes devem ser orientadas no sentido de que:

- Elas continuam apresentando risco de El;

- Devem manter uma boa higiene oral, incluindo avaliações odontológicas de rotina. Além disso, devem ser ensinadas a reconhecer os sinais e sintomas de $\mathrm{EI}$, e procurarem recurso médico com a maior brevidade possível quando houver suspeita da doença ${ }^{19}$.
10. Siu SC, Colman JM. Heart disease and pregnancy. Heart. 2001; 85: 710-5.

11. Harrison JL, Hoen B, Prendergast BD. Antibiotic prophylaxis for infective endocarditis. Lancet. 2008; 371: 1317-9.

12. Wolf JS, Bennet CJ, Dmochowski RR, Hollenbeck BK, Pearles MS, Schaeffe AJ. Best practice policy statement on urologic antimicrobial prophylaxis. J Urol. 2008; 179: 1379-90.

13. Hofmeyr GJ, Smaill FM. Antibiotic prophylaxis for cesarean section. Cochrane Database Syst Rev. 2002; (3): CD 000933

14. Costantine MM, Rahman M, Ghulmiyah L, Byers BD. Timing of perioperative antibiotics for cesarean delivery: a metaanalysis. Am J Obstet Gynecol. 2008; 199: 301.e1-301.e6

15. Stumpf PG. Practical solutions to improve safety in the obstetrics/gynecology office setting and in the operating room. Obstet Gynecol Clin North Am. 2008; 35: 19-35.

16. Prevention of infective endocarditis: guidelines from the American Heart Association: A guideline from the American Heart Association, Rheumatic Fever, Endocarditis and Kawasaki Disease Committee, Council on Cardiovascular Disease in the Young, and the Council on Clinical Cardiology, Council on Cardiovascular Surgery and Anesthesia, and the Quality of Care and Outcomes Research Interdisciplinary Working Group. J Am Dent Assoc. 2008; 139: 3-24.

17. Guidelines on prevention, diagnosis and treatment of infective endocarditis The Task Force on infective endocarditis of the European Society of Cardiology. Eur Heart J. 2004; 25: 267-76.

18. Embil JM, Chan KL. Canadian Cardiovascular Society Commentary. The American Heart Association 2007 endocarditis prophylaxis guidelines: a compromise between science and common sense. Can J Cardiol. 2008; 24: $673-5$.

\section{3 - Profilaxia do tromboembolismo}

O tromboembolismo venoso (TEV) é a principal causa de morte relacionada à gestação nos países desenvolvidos ${ }^{1}$. A gravidez, pelas modificações sobre a fisiologia da mulher, é causadora da tríade de Virchow, responsável pela trombose: hipercoagulabilidade, estase venosa e dano vascular ${ }^{2}$. Ocorre aumento do fibrinogênio, dos fatores pró-coagulantes I, II, VII, VIII, IX, XII, redução funcional de anticoagulantes naturais (proteína $S$ total e livre e proteína $\mathrm{C}$ ativada) e diminuição da fibrinólise, com o aumento do PAI I e II ${ }^{3}$. A estase venosa é consequência do

aumento da capacitância venosa provocada pela secreção de progesterona e da compressão do útero sobre o sistema venoso intra-abdominal.

A gestação já é um fator de risco (FR) para TEV, aumentando em 10 vezes, se comparado a não gestantes com mesma idade, e a idade acima de 35 anos aumenta o risco em 38\%. Algumas mulheres com FR pré-existentes não têm seu risco aumentado além do da gestação, devido à associação de FR transitórios, como desidratação, hiperemese, repouso $>4$ dias, infecções, parto cesáreo, hemorragias, gestação multifetal, entre outros ${ }^{4,5,6}$. 
As cardiopatias, quando com insuficiência cardíaca decompensada, são FR independentes, aumentando o risco de tromboembolismo em 8 vezes $^{7}$. Outros FR que aumentam muito o risco de TEV são: história prévia de tromboembolismo não relacionada a trauma, ou relacionadas a gestações prévias ou ao uso de anticoncepcional, trauma com imobilização prolongada, obesidade mórbida, síndrome antifosfolipídio e as trombofilias hereditárias ${ }^{8}$. Ainda é um desafio determinar quais pacientes devem receber profilaxia, e não há ensaios clínicos randomizados sobre a profilaxia de TEV na gestação, existindo vários esquemas encontrados na literatura. As condutas são feitas por orientação de experts, baseadas em estudos observacionais $^{6}$ (ver cap. 15).

\section{Recomendações gerais}

1) Toda mulher deve ter definido seu perfil de risco para TEV no início da gestação, sendo reavaliado quando internada em hospital ou quando com a associação de outros problemas intercorrentes que aumentem o risco ${ }^{4,5}$. As consideradas de alto risco devem iniciar a profilaxia já no primeiro trimestre ${ }^{9}$.

2) Evitar desidratação e minorar o repouso prolongado ${ }^{4,5}$ (IlaC).

3) Indicar uso de meia elástica compressiva (abaixo dos joelhos) durante a gestação e de até 6 a 12 semanas após o parto em pacientes com TEV prévia ou trombofilia ${ }^{4,5}$ (IlaC). Essa medida reduz o risco da síndrome pós-trombótica em pacientes com TVP aguda ou prévia ${ }^{10}$.

4) Considerar o uso de compressão pneumática intermitente durante e após parto cesáreo ${ }^{8}$.

5) Punção caudal para anestesia regional não deve ser realizada até 12 horas após a última dose de HNF ou HBPM SC em minidose $e^{4,5}$ e 24 horas após, quando utilizada em dose ajustada ${ }^{11}$, para evitar risco de hematoma epidural. A profilaxia deve ser reiniciada 6 horas após a retirada do cateter peridural ${ }^{4,5}$.

6) Aspirina isolada não é recomendada para profilaxia de TEV em nenhuma situação ${ }^{12}$ (IIIA).

\section{Esquemas de profilaxia anticoagulante ${ }^{4,5,8,10,13,14,15}$}

HNF: Minidose

$5.000 \cup \mathrm{SC} 12 / 12 \mathrm{~h}$ no $1^{\mathrm{o}}$ trimestre

$7.500 \cup \mathrm{SC} 12 / 12$ h no $2^{\circ}$ trimestre

10.000 U SC 12/12h no 3을 trimestre

Dose ajustada - Com controle do TTPA coletado no meio do intervalo entre as doses (sexta hora), mantendo-o entre 1,5 a $2,5 x$ o basal.

$$
\begin{array}{cccc}
\text { HBPM: Minidose } & & & \\
& \text { Peso } & \text { Peso } & \text { Peso } \\
& <50 \mathrm{~kg} & =51-90 \mathrm{~kg} & >90 \mathrm{~kg} \\
\text { Enoxaparina } \rightarrow & 20 \mathrm{mg} \mathrm{SC} & 40 \mathrm{mg} \mathrm{SC} & 40 \mathrm{mg} \mathrm{SC} \\
& 24 / 24 \mathrm{~h} & 24 / 24 \mathrm{~h} & 12 / 12 \mathrm{~h} \\
\text { Dalteparina } \rightarrow & 2.500 \mathrm{USC} & 5.000 \mathrm{UC} & 5.000 \mathrm{SC} \\
& 24 / 24 \mathrm{~h} & 24 / 24 \mathrm{~h} & 12 / 12 \mathrm{~h}
\end{array}
$$

Dose ajustada:

Enoxaparina $\rightarrow \quad 1 \mathrm{mg} / \mathrm{kg} \mathrm{SC} 12 / 12 \mathrm{~h}$ ou antifator Xa entre 0,5 e $1,2 \mathrm{UI} / \mathrm{ml}$

Dalteparina $\rightarrow \quad 100 \mathrm{U} / \mathrm{kg} \mathrm{SC} 12 / 12 \mathrm{~h}$

Cumarínicos: dose ajustada: INR entre 2 e 3 (ver cap. 6).

\section{Recomendações para tromboprofilaxia na gestação}

1) TEV prévia associada a FR transitório não mais presente - Acompanhamento clínico pré-parto e anticoagulação dose ajustada pós-parto por 6 a 12 semanas (IC).

2) TEV prévia idiopática - Acompanhamento clínico ou heparina minidose até o parto e um dos anticoagulantes dose ajustada pós-parto por 6 a 12 semanas (IlaC).

3) TEV prévia com comprovada trombofilia ou história familiar de trombose - Minidose de uma das heparinas até o parto e um dos anticoagulantes dose ajustada pós-parto por 6 a 12 semanas (IlaB).

4) TEV prévia e deficiência de antitrombina, ou combinação de heterozigose para fator $V$ Leiden com heterozigose para mutação da protrombina, ou homozigose de uma delas Anticoagulação dose ajustada antes e depois do parto (IlaC).

5) Vários episódios prévios de TEV ou em uso crônico de anticoagulante - Manter dose ajustada antes e após parto (IlaC).

6) Outras trombofilias, mas sem história prévia de TEV - Acompanhamento clínico ou heparinas em minidose antes do parto e um dos anticoagulantes em dose ajustada pósparto (IlaC).

7) Pacientes com três ou mais FR persistentes (exceto TEV prévia e/ou trombofilia) - Heparinas em minidose antes do parto e de 3 a 5 dias pós-parto. As heparinas, quando usadas em minidose periparto, devem ser iniciadas 12 horas antes e repetidas a cada 24 horas, se HBPM, ou 12 horas, se HNF (IlaC).

8) Pacientes com dois FR persistentes - Uma das heparinas em minidose por 3 a 5 dias pós-parto, mesmo que seja vaginal (IlaC).

9) Doença reumática com fibrilação atrial (FA), insuficiência cardíaca descompensada com ou sem FA, trombo intracavitário, estenose mitral grave com átrio esquerdo aumentado (> 50 $\mathrm{mm}$ ) ou contraste espontâneo ao ecocardiograma, cardiopatia congênita cianótica complexa - Anticoagulação dose ajustada contínua (IlaB).

\section{Referências}

1. Simpson EL, Lawrenson RA, Nightingale AL, Farmer RD. Venous thromboembolism in pregnancy and the puerperium: incidence and additional risk factors from a London database. BJOG. 2001; 108: 56-60.

2. Andres RL, Miles A. Venous thromboembolism and pregnancy. Obstet Gynecol Clin North Am. 2001; 28: 613-30. 
3. Clark P, Brennand J, Conkie JA, McCall F, Greer IA, Walker ID. Activated protein $\mathrm{C}$ sensivity, protein $\mathrm{C}$, protein $\mathrm{S}$ and coagulation in normal pregnancy. Thromb Haemost. 1998; 79: 1166-70.

4. Royal College of Obstetricians and Gynaecologists. Thromboembolic disease in regnancy and the puerperium: acute management. [Acessed 2008 Jun 10] Available from: http://www.rcog.org.uk/womens-health/clinical-guidance/ thromboembolic/disease

5. Royal College of Obstetricians and Gynaecologists. Thromboprophylaxis during pregnancy, labor and after vaginal delivery. [Acessed 2008 Jun 10]. Available from: http://www.rcog.org.uk/womens-health/clinical-guidance/ thromboprophylaxis

6. James AH, Jamison MG, Brancazio LR, Myers ER. Venous thromboembolism during pregnancy and the postpartum period: incidence, risk factors, and mortality. Am J Obstet Gynecol. 2006; 194: 1311-5.

7. Tedoldi CL, Bandinelli E, Menna Barreto SS, Manfroi WC. Heart failure as a strong risk factor for venous thromboembolism in pregnancy. In: Meeting of the World Congress of Cardiology. Proceedings of the World Congress of Cardiology; 2006 Sept 2-6; Barcelona (Spain). Eur Heart). 2006; 27 (Abstract Suppl): 296.
8. Greer IA. Thrombosis in pregnancy: maternal and fetal issues. Lancet. 1999; 353: $1258-65$.

9. James AH, Tapson VF, Goldhaber SZ. Thrombosis during pregnancy and the postpartum period. Am J Obstet Gynecol. 2005; 193: 216-9.

10. SOGC Clinical Practice Guidelines. Prevention and treatment of venous thromboembolism in obstetrics. J Soc Obstet Gynaecol Can. 2000; 22 (9): 736-42.

11. Bates SM, Greer IA, Hirsh J, Ginsberg JS. Use of antithrombotic agents during pregnancy. Chest. 2004; 126: 627S-644S

12. Geets WH, Pineo GF, Heit JA, Bergqvist D, Lassen MR, Colwell CW, et al. Prevention of venous thromboembolism. Chest. 2004; 126: 338S-400S.

13. Ginsberg JS, Bates SM. Management of venous thromboembolism during pregnancy. J Thromb Haemost. 2003; 1: 1435-42.

14. Elkayam U, Bitar F. Valvular heart disease and pregnancy part I: native valves. J Am Coll Cardiol. 2005; 46: 223-30.

15. Blann AD, Landray MJ, Lip GYH. ABC of antithrombotic therapy: an overview of antithrombotic therapy. BMJ. 2002; 325: 762-5.

presente nos casos refratários ao tratamento clínico ou na impossibilidade do tratamento percutâneo por cateter balão. As causas mais frequentes são: insuficiência cardíaca, endocardite infecciosa, trombose de prótese valvar e dissecção de aorta ${ }^{4,5,6}$.

Os riscos relacionados ao procedimento cirúrgico não são diferentes aos de mulheres não grávidas. Contudo, a morbidade e mortalidade materna têm sido relatadas como maiores na gestação ${ }^{6,7,8}$, porque geralmente a cirurgia cardíaca é indicada em situações de extrema gravidade. Em dois serviços de referência a mortalidade materna variou de $8,6 \%^{7}$ a $13,3 \%^{6}$, sendo a insuficiência cardíaca refratária a principal indicação e a comissurotomia mitral o procedimento mais frequente $\mathrm{e}^{6,7}$.

O prognóstico fetal está relacionado com a vitalidade fetal prévia ao procedimento, ao tempo e à temperatura da circulação extracorpórea e à idade gestacional no momento da cirurgia. Em nosso país, a mortalidade fetal é descrita como variando de $18,6 \%^{7}$ a 33,3\% ${ }^{6}$. Recomendações durante o procedimento para melhor resultado obstétrico e fetal (IlaC):

- Normotermia ${ }^{4,5,6,9}$;

- Alto fluxo na circulação extracorpórea ${ }^{5}$;

- Fluxo pulsátil ${ }^{4,6,9}$;

- Pressão arterial média $>60 \mathrm{mmHg}^{4}$;

- Uterolítico profilático;

- Cardiotocografia contínua ${ }^{5,6,9}$;

- Dopplerfluxometria;

- Manter hematócrito acima de 25\% .

\section{3 - Cardioversão elétrica}

Citânia Lúcia Tedoldi

Cardioversão elétrica sincronizada pode ser necessária em pacientes com taquiarritmias supraventriculares ou ventriculares que não respondem ao uso de drogas ou quando desenvolvem instabilização hemodinâmica. O

A indicação de cirurgia cardíaca na gravidez está 